\title{
Humidity influence on the adhesion of SU-8 polymer from MEMS applications
}

\author{
Corina Birleanu ${ }^{1, *}$, Marius Pustan ${ }^{1}$, Rodica Voicu $^{2}$, Florina Serdean ${ }^{1}$ and Violeta Merie ${ }^{1}$ \\ ${ }^{1}$ Technical University from Cluj-Napoca, Micro and Nano System Laboratory, 400641, B-dul Muncii \\ 103-105, Romania \\ ${ }^{2}$ Laboratory of Modeling, Simulation and CAD, National Institute for R\&D in Microtechnologies - \\ IMT Bucharest, 126A Erou Iancu Nicolae Street, 077190, Bucharest, Romania
}

\begin{abstract}
In this paper, the adhesion behaviors of SU-8 polymer thin film from MEMS application were investigated as a function of relative humidity. The adhesion test between the AFM tip and SU-8 polymer have been extensively studied using the atomic force microscope (AFM), for a relative humidity (RH) varying between 20 and $90 \%$. The samples for tests are SU-8 polymers hard baked at different temperatures. The hard bake temperature changes the tribo-mechanical properties of polymers. The paper reports the measurements and the modeling of adhesion forces versus humidity in controlled ranges between 20 to $90 \% \mathrm{RH}$. To investigate the effect of relative humidity on adhesion for SU-8 polymer hard baked we used an analytical method which encompasses the effect of capillarity as well as the solid-to-solid interaction. While the capillary force expression is considered to be the sum of the superficial tension and the Laplace force for the solid-solid interaction is expressed by the Derjagin, Muller and Toropov (DMT) model of solids adhesion. The analytical results obtained are in accordance with those obtained experimentally.
\end{abstract}

\section{Introduction}

Microsystems field is one of the most interesting and exciting fields that present interest in science and technology today. Many physical phenomena that are neglected at macro scale acquire dominant characteristics at micro and nano scale. These phenomena exploited in an intelligent way lead to the manufacture of Micro-ElectroMechanical-Systems (MEMS) devices with operation principles completely different than the macro scale counterpart system [1].

These micro-scale devices are commonly made from silicon ( $\mathrm{Si}$ ) or some cross-linked polymers such as SU-8. Though Si has many attractive properties related to hardness, stiffness, fatigue and the ease of mass-fabrication by the semiconductor fabrication technology, $\mathrm{Si}$ is very poor in tribological performances [2]. In a dynamic state when silicon is in contact with itself or other similar material, it exhibits high friction, high initial and dynamic friction, and considerable wear [2]. The main failure mechanisms in MEMS are stiction, friction and wear. When two surfaces are close to each other, the attractive

\footnotetext{
${ }^{*}$ Corresponding author: Corina.Barleanu@,omt.utcluj.ro
} 
surface forces (capillary, van der Waals and electrostatic forces) play an important role in device performance $[2,3]$.

SU-8 is an epoxy-based negative photoresist material near UV photoresist based on EPON SU-8 epoxy resin that has been first developed and patented it compositions by IBM as far back as 1989 [4, 5]. Besides the many advantages of SU-8 photoresists over available UV photoresists, an import advantage is its use in the fabrication of MEMS. Because of its aromatic functionality and highly cross-linked matrix, the SU-8 resist is thermally stable and chemically very inert [6].

Moreover, the SU-8 polymer is insoluble in water, biocompatible and after polymerization has chemical durability. Also SU-8 polymer allows to obtain surfaces with the very best aspects of roughness, which is a basic need for designing reliable functional MEMS mechanical structures with good mechanical and tribological properties [7]. The SU-8 polymer parameters and data for the mechanical and tribological properties published in the literature vary substantially as a function of the processing conditions, testing method as well as the operating parameters [8].

In this paper, using an AFM equipped with an environment chamber, the nano adhesion behaviors of SU-8 polymer hard baked at different temperatures were systematically studied as functions of relative humidity, between $20-90 \% \mathrm{RH}$ and the corresponding mechanisms were discussed. A full understanding of the adhesion behaviors of SU-8 polymer is an essential issue and this investigation can provide further insights into minimizing the potential failure of MEMS in humid environment. The experimental tests are performed under different testing condition in order to investigate the humidity effect on the adhesion phenomena of such MEMS material.

\section{Materials and methods}

\subsection{Thin-film preparation}

The SU-8 material used in this paper as testing material is a negative, epoxy-type, near-UV (350-400 nm) photo-resist in which eight epoxy groups are attached to a single molecule.

Silicon (100) wafers were used as substrates for the SU-8 polymer films.

SU-8 2015 polymer (Micro-Chem) was spin on the wafers using a spinner with thicknesses of around $10 \mu \mathrm{m}$. The SU-8 samples were hard baked at different temperatures to complete the crosslinking of the polymer. Four samples of SU-8 polymers were selected for experimental characterizations with different hard bake temperature such as: $125^{\circ} \mathrm{C}$, $165^{\circ} \mathrm{C}, 195^{\circ} \mathrm{C}$ and $215^{\circ} \mathrm{C}$. The final film thickness was about $10 \mu \mathrm{m}$. These samples are experimentally investigated under different testing humidity in order to determine their adhesion behavior.

The experimental result for contact angle $\theta$ of water vapor on the SU- 8 surface was $65^{\circ}$ $\pm 4^{\circ}$ at $22^{\circ} \mathrm{C}$ ambient conditions. Contact angle measurements were made with a Theta Lite Optical Tensiometer TL 101 with a USB 2 digital camera. Contact angles were determined with OneAttension software by sessile drop method using Young-Laplace. Five measurements were made on surface under investigation.

\subsection{Atomic force microscopy}

The structural and surface morphology and adhesion forces between SU-8 samples and AFM (Atomic Force Microscopy) cantilever were determined at different relative humidity varying from 20 to $90 \%$. 
Surface roughness measurements were conducted by atomic force microscopy using AFM - XE 70 in tapping mode, to avoid the scratch and deterioration of the polymeric surface. The AFM probe used in the surfaces characterization is the PPP-NCHR AFM probe with unique features like: highly doped silicon to dissipate static charge, Al coating on detector side of cantilever, with the following parameters: the thickness is $4 \mu \mathrm{m}$, the width is equal to $30 \mu \mathrm{m}$, the length is $125 \mu \mathrm{m}$, the force constant is $42 \mathrm{~N} / \mathrm{m}$ and the resonant frequency is $330 \mathrm{kHz}$.

The adhesive effect between the AFM probe $\left(\mathrm{Si}_{3} \mathrm{~N}_{4}\right)$ and the samples were evaluated using the force spectroscopy mode of the apparatus, the so-called force-displacement measurements, in a controlled environment (clean room). The tip used was $\mathrm{Si}_{3} \mathrm{~N}_{4} \mathrm{AFM}$ tip HQ - NSC 35/Hard/Al BS with a nominal value of the spring constant $\mathrm{k}=16 \mathrm{~N} / \mathrm{m}$ and radius $<20 \mathrm{~nm}$. The load applied for all measurements was $50 \mathrm{nN}$. The set point was $10 \mathrm{nN}$ while the scan rate was $0.75 \mathrm{~Hz}$. Measurements were performed over a range of processing RH between 10 to $90 \%$ and a contact time up to a few seconds.

Such measurements provide correct information about the surface energy and the adhesion only if the contact between the AFM tip and the cantilever surface remains in the elastic range. Literature indicates that at $20^{\circ} \mathrm{C}$ and $35 \% \mathrm{RH}$, when the load is varied between $20 \mathrm{nN}$ and $250 \mathrm{nN}$, there is no significant change in the adhesion force [9].

For all experimental investigations, the AFM was enclosed in an Environmental Chamber (Glove Box). Relative humidity within the glovebox was controlled by a Humidity Control System. The water used to humidify the chamber was distilled and deionized. Humidity levels were monitored continuously by a radiosonde. Prior to measurement, the humidity was allowed to reach equilibrium as indicated by a constant value. After that, going from low to high humidity and vice versa to dry conditions was achieved in less than $10 \mathrm{~min}$. The relative humidity is changed, from $10 \%$ up to $90 \%$ in steps of $10 \%$. The air flow did not significantly affect AFM operation and all the common adjustments were readily accessible via the glove ports. Operating over extended humidity of $80 \% \mathrm{RH}$ was avoided because of the possible damage to the AFM scanner. Each measurement was repeated many times in order to improve the accuracy of the experimental results and the mean value was computed.

\section{Results and discussions}

The paper is motivated by tribological problems observed in MEMS / NEMS devices. It is postulated that a fundamental understanding of nanoscale tribological behaviour can create guidelines for the design and manufacture of reliable MEMS devices.

\subsection{Topography}

To analyse the adhesion force between SU-8 surface and AFM tip it is important to measure the roughness value of the contact surfaces.

For evaluating the surface topography of the samples was utilized the Non-Contact AFM mode (NC-AFM) based on the attractive inter-atomic force between the tip and the sample surface. In AFM, a sharp tip at the end of a cantilever is vibrated near the surface of a sample and was scanned over $3 \mu \mathrm{m} \times 3 \mu \mathrm{m}$ surface area.

Surface roughness was evaluated by flattening the images (second order). A secondorder polynomial plane correction was carried out in order to reduce waviness due to scanner bow and variations of the film thickness. Then a line-wise leveling was performed to reduce a possible line-by-line repetition error. As surface roughness parameters we qualified the root mean square (RMS or $R_{q}$ ) surface roughness provided by the XEI software. $R_{q}$ is more sensitive to occasional highs and lows, making it a valuable 
complement to the arithmetic average height of roughness-component irregularities from the mean line $R_{a}$. For instance, the acquirements of AFM images of morphologies surface and roughness surface for upper side of SU-8 sample surface hard baked at $125^{\circ} \mathrm{C}$ is the $\mathrm{R}_{\mathrm{q}}$ $=0.409 \mathrm{~nm}$ and $\mathrm{R}_{\mathrm{a}}=0.325 \mathrm{~nm}$.

The AFM images for each of the contact surfaces, together with the statistical parameters provided for roughness by the software used for interpreting the experimental data (XEI Image Processing Tool for SPM) are given in Fig. 1 (a) and (b).
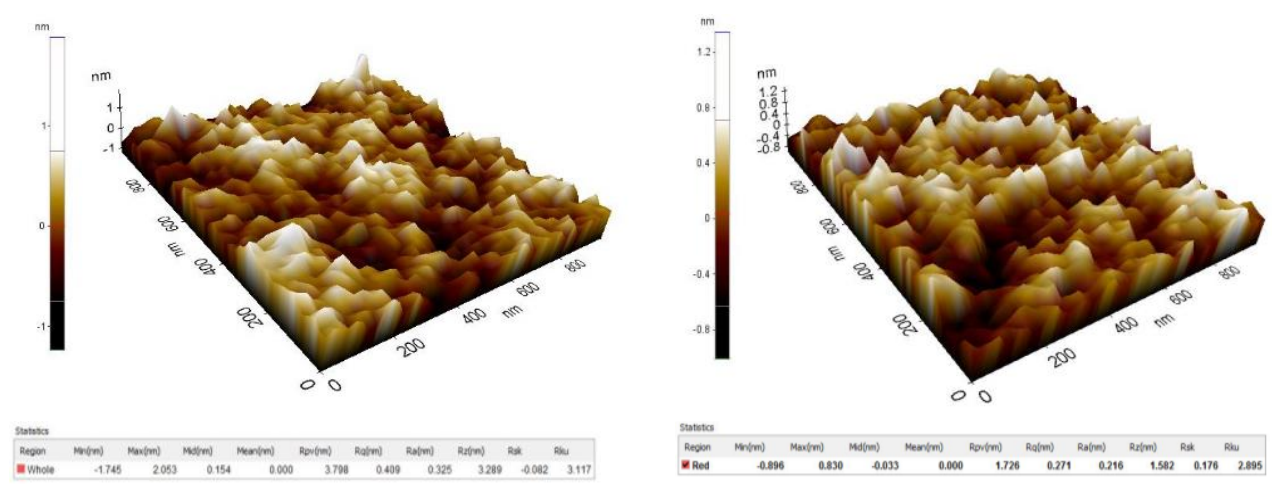

Fig. 1. Topographic images in NC-AFM mode of (a) SU-8 hard baked at $125^{\circ} \mathrm{C}$; (b) SU-8 hard baked at $215^{\circ} \mathrm{C}$.

The roughness parameters of investigated of SU-8 polymers decrease as the hard bake temperature is increased from $125^{\circ} \mathrm{C}$ to $215^{\circ} \mathrm{C}$. The $\mathrm{R}_{\mathrm{a}}$ roughness is $0.325 \mathrm{~nm}$ for $\mathrm{SU}-8$ polymer hard baked at $125^{\circ} \mathrm{C}$, it decreases to $0.296 \mathrm{~nm}$ for $165^{\circ} \mathrm{C}$ hard baked temperature, 0.268 for a hard bake temperature of $195^{\circ} \mathrm{C}$ and 0.216 for the polymer baked at $215^{\circ} \mathrm{C}$.

Reproducible roughness measurements require a well-defined set of operating conditions and parameters such as the AFM peak radius, pixel size, scanning speed, and width. In order to compare the results obtained between different batches of samples or different laboratories, it is important to know these parameters.

\subsection{Adhesion force}

\subsubsection{Experimental evaluation of adhesion force.}

The experimental determination of the pull-off forces between the AFM tip and SU-8 surface at different hard bake temperature was conducted using the spectroscopy in point mode of the AFM.

Figure 2 shows the pull-off forces measured for different humidity values for the SU-8 thin film with at different hard bake temperature at ambient temperature, $22^{\circ} \mathrm{C}$. Each reported value is the average of five measurements taken at different regions on the surface of each sample.

Based on such experimental investigations as those in figure 2, the values of the adhesion force for the SU- 8 hard baked at $125^{\circ} \mathrm{C}$ and $215^{\circ} \mathrm{C}$ investigated at different humidity are shown in figure 3.

The increase of the pull-off forces with the $\mathrm{RH}$ is more pronounced in the $30 \%$ to $70 \%$ range. For a RH less than $30 \%$ the thickness of the water film on the SU-8 surface is too thin to form a capillary neck with the tip asperity. At around $70 \% \mathrm{RH}$, the adhesion force shows a maximum value, but if the RH is further increased the pull-off force shows a decrease. When the water film reaches a minimum required thickness at $30 \% \mathrm{RH}$, a water 
bridge forms between the asperity and the surface, thus leading to a sudden increase of the capillary force. Binggeli and Mate [10] explained that the decrease in adhesion force at high relative humidity as a result of the interactions between capillary forces and forces related to the chemical bonding of the water molecules [11]. As a result, one can distinguish between three regimes of adhesion force versus humidity: a regime dominate by solid-tosolid interaction, van der Walls regime at low $\mathrm{RH}(\mathrm{RH}<30 \%)$, a regime dominated by the capillary force $(30 \%<\mathrm{RH}<70 \%)$ and a high humidity regime $(\mathrm{RH}>70 \%)$.
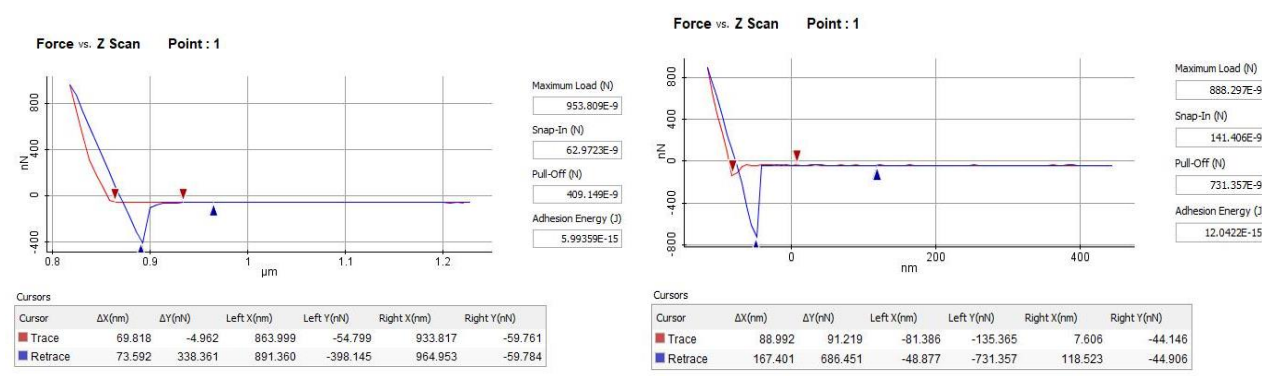

Fig. 2. Pull-off force between AFM tip and SU-8 hard baked at $125 \mathrm{oC}$ and test at $22 \mathrm{oC}$ and different RH values: $20 \%$ and $60 \%$.

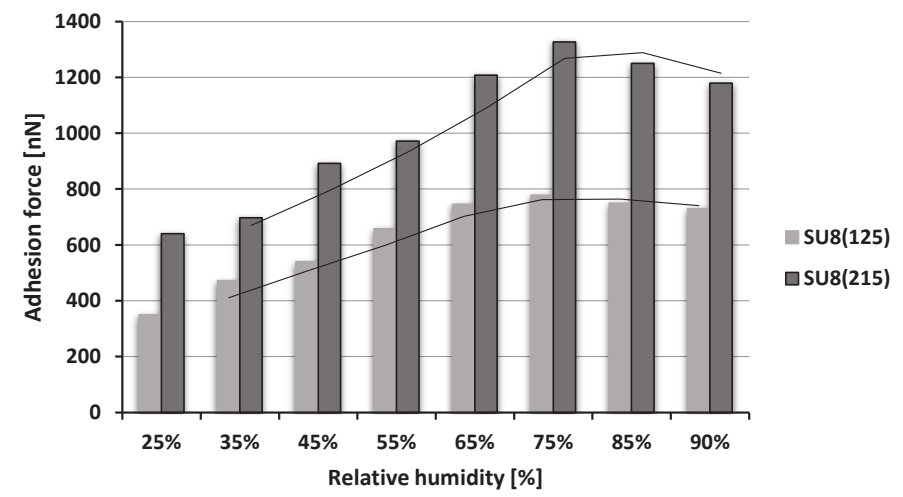

Fig. 3. Adhesion forces versus relative humidity [RH\%] measured between the SU-8 hard baked at $125^{\circ} \mathrm{C}$ and $215^{\circ} \mathrm{C}$ and $\mathrm{AFM}$ tip.

Increasing humidity generally promotes increases water adsorption leading to increased contributions from capillary bridges between the tip and SU-8 thin film surface towards the pull-off force. During measurements of the pull-off force no deteriorations in surface samples were observed and there was no significant change in the radius on the AFM tip. Therefore, we can say that solid adhesion component is the main parameter contributing to the pull-off force.

\subsubsection{Analytical interpretation of adhesion force}

In the presence of an annulus of pure liquid the adhesion force $P_{a d h}$ can be written taking into account the influence of four attractive forces acting, which are van der Waals force, electrostatic force, capillary force and others.

$$
P_{a d h}=P_{v d w}(d)+P_{c a p}(\Delta p)+P_{t e n}\left(\lambda_{L V}\right)+P_{\text {others }}
$$


Where: $P_{v d w}(d)$ is the force due to van der Walls interaction with separation $d$ between surfaces; $P_{c a p}(\Delta p)$ is the capillary force based on Laplace pressure $(\Delta p) ; P_{\text {ten }}$ $\left(\gamma_{L V}\right)$ is the force due to liquid-vapor surface tension $\left(\gamma_{L V}\right)$ and other interactions $P_{\text {others. }}$ In most practical cases, when a capillarity bridge was formed the capillary force can be expressed as:

$$
P_{c a p}(\Delta p)=4 \pi R \gamma_{L V}
$$

Most experimental results indicate that predominant mechanism of adhesion is given by van der Walls forces, solid-to-solid interactions. Recent research shows that capillary forces are a factor that contributes to the phenomenon of adhesion. Capillary forces created by natural air humidity further increase the adhesion force [12].

The van der Waals forces $\left(P_{v d w}\right)$ which act between two surfaces and result from the interaction between the instantaneous dipole moments of atom are given by $[13,14]$ :

$$
P_{v d w}(d)=\frac{A R}{6 d^{2}}=4 \pi R \gamma_{S L}
$$

where: $A$ is the Hamaker constant of the liquid, $d$ is the minimum distance between surfaces and $\gamma_{S L}$ is the solid-liquid surface tension.

The force due to the other interactions $P_{\text {others }}$ can be neglected.

Assuming that both asperities of the contact surfaces are spherical, a single asperity contact occurs when the adhesive force acting on the asperity deforms it by at least the RMS roughness and is close to the DMT model. Therefore, we applied the DMT theory for the contact between solids. The surfaces in contact are assumed to be rigid and the adhesion is estimated based on folowing equation:

$$
P_{a d h}^{D M T}=-2 \pi W_{a d h} R
$$

In this equation $R$ is the reduced radius of contact, which is calculated as $R=\left(1 / R_{1}+1 / R_{2}\right)^{-1} ; R_{1}$ and $R_{2}$ are radii of contacting surfaces; $R_{1}=18 \mathrm{~nm}$ and $R_{2}=$ $0.304 \mathrm{~nm}$. Because the differences between the two rays are significant, more than 18 times in the calculations $\mathrm{R}$ are considered to be equal with AFM probe radius $R_{1}$ (18 $\mathrm{nm})$. The work of adhesion of two flat surfaces $W_{\text {adh }}$ can be calculated using the following equation [15]:

$$
W_{a d h}=-\frac{A}{12 \pi d^{2}}
$$

A is Hamakar constant which depends on the properties of the two materials and the intervening medium. Typical value for A for SU-8 and AFM tip typically made of silicon nitride are $A_{\text {air }}=6.5 \times 10^{-21} \mathrm{~J}$ in the air [16] and $A_{\text {water }}=1.8 \times 10^{-21} \mathrm{~J}$ in the water [17]. In this case when two surfaces are in contact in a humidity atmosphere the absorbed and condensed water molecules are present between these surfaces separated at a gap with thickness of molecular distance.

The work of adhesion of the two surfaces in contact separated by an atomic distance $d \approx$ $0.4 \mathrm{~nm}[15]$ is approximately equal to $-43.1 \mathrm{~mJ} / \mathrm{m}^{2}$ in the air and $-11.93 \mathrm{~mJ} / \mathrm{m}^{2}$ in the water.

Assuming tip radius $R$ is $18 \mathrm{~nm}$, the DMT adhesion forces in the air and the water for a single contact based on (6) and (16) are $P_{D M T}^{\text {air }}=487.45 n N$ and $P_{D M T}^{\text {water }}=134.92 n N$, respectively. If the humidity increases from 0 to $100 \%$ the DMT adhesion force will take a value between $P_{D M T}^{a i r}$ and $P_{D M T}^{\text {water }}$. For an intermediary humidity will be used the Wan et al. 
[18] approximation method. The total adhesion force based on analytically interpretation is calculated as the sum of DMT and capillary adhesion.

To take into account the analytical capillarity contributions to pull-off forces for SU-8 samples, the contact angler for the SU-8 surface and AFM tip is set as a value of $\theta=65^{\circ}$. The contact radius $R=18 \mathrm{~nm}$, the ambient temperature $T=22^{\circ} \mathrm{C}$, the surface tension $\gamma_{\mathrm{LV}}=$ $73 \mathrm{~mJ} / \mathrm{m}^{2}$ and molecular volume of water $V=1.8 \times 10^{-5} \mathrm{~m}^{3} / \mathrm{mol}[14]$.

In figure 4 is presented the variation of pull-off forces, capillary forces, surface tension forces and solid-to-solid forces based on DMT model in relation with relative humidity, RH.

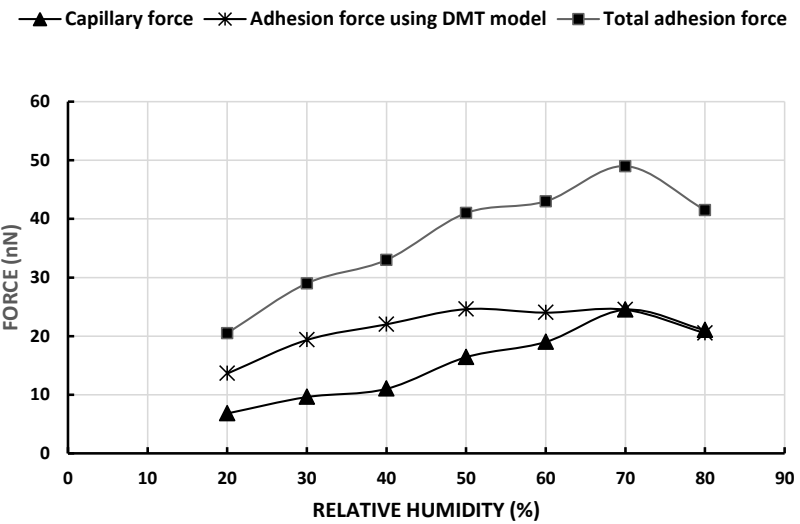

Fig. 4. The variation of the capillary force, the adhesion force based on DMT model force and the total adhesion force with the relative humidity for a single contact between the SU-8 hard baked at $125^{\circ} \mathrm{C}$ and AFM tip.

The total capillary force increases to the $24.5 \mathrm{nN}$ at $70 \%$ relative humidity. The DMT adhesion force decreases with an increase a relative humidity. The DMT adhesion force occupies a large value in total adhesion. The total adhesion force increases slowly to $49 \mathrm{nN}$ at a value of relative humidity of $70 \%$.

Due to the force applied at the thin film from SU-8, the contact between the tip and the surface is along a line segment. Studying the contact line segment, it was established that there were 15 contact points which were modelled as the contact between the sphere and a plane due to the roughness of SU-8 and AFM tip.

Then the total adhesion was computed according to [19] and the results are presented in Fig. 5.

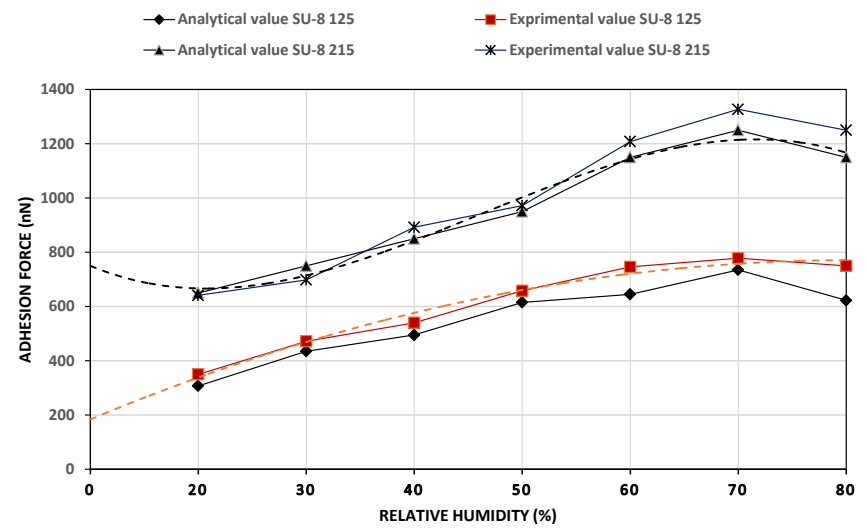

Fig. 5. Comparison of the analytical results with the measurement data for whole line contact between the AFM tip and SU-8 hard baked at $125^{\circ} \mathrm{C}$ and $215^{\circ} \mathrm{C}$, respectively. 


\section{Conclusions}

This paper presents the influence of the relative humidity on the values of the pull off forces under normal loading based on analytical and experimental studies in the case SU-8 polymers hard baked at different temperatures. The salient conclusions arising from this work are summarized below:

Accurate determination of pull-off forces at the molecular level leads to a significant insight into the areas of interest such as SU-8 material.

In order to investigate the effect of capillarity due to the presence of humidity range on adhesion (pull-off forces), we considered the capillarity force consisting of the Laplace force and the surface tension force and the solid-to-solid interaction by DMT theory, starting from a single contact. In [20] is suggested that single asperity contact occurs when the adhesive force acting on the asperity deforms it by at least the RMS roughness. Then, using the model proposed by the authors in [19] the results were extrapolated to the entire length of contact between AFM tip and samples. In interpreting the data presented in the ambient temperature at different values of relative humidity it was necessary to assume a value for Kelvin radius as $0.4 \mathrm{~nm}$ and to establish the equilibrium separation.

In conclusion, we used the AFM investigation to determine the pull-off forces on a nanostructure as SU-8 samples. The capillarity forces theory and analytical model used for adhesion forces components gives results which are in good agreement with the experimental data.

This work was supported by a grant of the Romanian National Authority for Scientific Research and Innovation, CCCDI - UEFISCDI, project number 22/2016, within PNCDI III, ERA-MANUNET-IIRobogrip project "Microgrippers as end-effectors with integrated sensors for microrobotic applications (ROBOGRIP)".

\section{References}

1. M. Pustan, G. Ekwinski, Z. Rymuza, Int J Mater Res, 98 (5) (2007)

2. N.B. Tay, M. Minn, S.K. Sinha, Tribol Lett, 2011.44 (2) (2011)

3. W.M. Van Spengen, R. Puers, I. De Wolf, J Adhes Sci Technol, 17 (4) (2003)

4. J. Gelorme, R. Cox, S. Gutierrez, US Patent No. 4,882,245. Washington, DC: US Patent and Trademark Office (1989)

5. R. Feng, R.J. Farris, J Micromech Microeng 13 (1) (2002)

6. R. Martinez-Duarte, M.J. Madou, Microfluidics and Nanofluidics Handbook (2011)

7. J Hammacher, A Fuelle, J Flaemig, J Saupe, Microsyst Technol, 14 (9-11) (2008)

8. J Lee, H Shin, S Kim, S Hong, J Chung, Jpn J Appl Phys 2003. 42 (10A) (2003)

9. Y. Ando, Wear, 238 (1) (2000)

10. M. Binggeli, C. Mate, Appl Phys Lett, 65 (4) (1994)

11. M Farshchi-Tabrizi, M Kappl, Y Cheng, J Gutmann, Langmuir, 22 (5) (2006)

12. T.W. Kim, B. Bhushan, J R Soc Interface, 5(20), (2008)

13. A Torii, M Sasaki, K Hane, Proceedings An Investigation of Micro Structures, Sensors, Actuators, Machines and Systems, IEEE (1993)

14. D. Xu, K.M. Liechti, K. Ravi-Chandar, J Colloid Interface Sci, 315 (2) (2007)

15. J.N. Israelachvili, Intermolecular and surface forces, Academic press (2011)

16. S. Matope, A. Van der Merwe, Y. Rabinovich, S. Afr. j. ind. eng, 24 (2) (2013)

17. H.-J. Butt, B. Cappella, M. Kappl, Surf Sci Rep, 59 (1) (2005)

18. K.T. Wan, D.T. Smith, B.R. Lawn, J Am Ceram Soc, 75 (3) (1992)

19. M. Pustan, C. Birleanu, C. Dudescu, Appl Surf Sci (2016)

20. D. Tabor, J Colloid Interface Sci, 58 (1) (1977) 